\title{
Evaluacion de compuestos fenolicos de (Citrus sinensis) y su capacidad antioxidante
}

\section{Evaluation of Phenolic Compounds (Citrus sinensis) and its Antioxidant Capacity}

\author{
Nelson Alfonso Vega Contreras ${ }^{1}$ y Martha Liliana torres Salazar ${ }^{2}$
}

\begin{abstract}
Resumen
La presente investigación tiene como objetivo principal evaluar los compuestos fenólicos extraídos de la cáscara de la naranja valencia (Citrus. sinensis), esto con la finalidad de ser aplicados en la industria cárnica como antioxidantes, y su posible aceptación sensorial, para lo cual se aplicaron dos métodos diferentes de extracción, siendo estos el ultrasonido y Soxhlet, utilizando el etanol como solvente, donde último el método resulto más eficiente para la extracción de los compuestos fenólicos, con un porcentaje de efectividad significativo, la identificación se realizó mediante cromatografía por HPLC/DAD, en la cual se detectaron compuestos como ácido galico, acido para hidroxibenzoico, ácido vanilico, ácido cafeico, acido $p$-cumarico, ácido felurico, entre otros, la determinación de la actividad antioxidante, se realizó por la decoloración del beta caroteno el cual decolora rápidamente sin la presencia de un antioxidante, la aplicación de los compuestos fenólicos se realizaron en productos embutidos (chorizos) crudos frescos, en donde se sometieron a una evaluación sensorial para mirar su posible aceptación lo que permitió concluir que las cascaras de Citrus Sinensis tiene una alta capacidad de inhibir la oxidación de los productos cárnicos.
\end{abstract}

Palabras clave: cítricos, extracción, flavonoides, inhibición, compuestos fenólicos

\begin{abstract}
The main objective of this research is to evaluate the phenolic compounds extracted from the peel of the Valencia orange (Citrus. sinensis), this with the purpose of being applied in the meat industry as antioxidants, and its possible sensory acceptance, for which two different extraction methods were applied, being these the ultrasound and Soxhlet, using ethanol as solvent, where the latter method was more efficient for the extraction of phenolic compounds, with a significant percentage of effectiveness, the identification was performed by chromatography by HPLC/DAD, in which compounds were detected as gallic acid, parahydroxybenzoic acid, vanillic acid, caffeic acid, p-coumaric acid, feluric acid, feluric acid, parahydroxybenzoic acid, vanillic acid, caffeic acid, p-coumaric acid, feluric acid, para-hydroxybenzoic acid and p-coumaric acid, Vanillic acid, caffeic acid, p-coumaric acid, feluric acid, among others, the determination of the antioxidant activity was carried out by the decolorization of beta carotene, which decolorizes rapidly without the presence of an antioxidant, the application of phenolic compounds was carried out in fresh raw sausages and sausage products, where they were subjected to a sensory evaluation to see their possible acceptance, which allowed concluding that Citrus Sinensis peels have a high capacity to inhibit the oxidation of meat products.
\end{abstract}

Keywords: citrus, extraction, flavonoids, Inhibition, phenolic compounds

Recepción: 04-feb-2021

Aceptación: 10-May-2021

\footnotetext{
${ }^{1}$ M.Sc. en prácticas pedagógicas, Esp. bioquímica Departamento medio ambiente, Universidad Francisco de paula Santander Cúcuta Colombia, Norte de Santander, Colombia. Autor de correspondencia: Nelavec6@gmail.com

${ }^{2}$ Ing. Agroindustrial, Departamento medio ambiente Universidad Francisco de Paula Santander Colombia
} 


\section{Introducción}

El cuerpo humano tiene la capacidad de generar sustancias antioxidantes que se encargan de contrarrestar el estado de estrés oxidativo [1], sin embargo, el consumo de alimentos ha sido recomendado debido al aporte nutricional y de biomoléculas que cuentan con capacidad antioxidante como los compuestos fenólicos y flavonoides [2], los antioxidantes en su gran mayoría los encontramos presentes en plantas, microorganismos, hongos $\mathrm{y}$ en algunos tejidos de animales, se ha comprobado que los antioxidantes presentes en las frutas y las verduras ayudan a la salud de las personas reduciendo el riesgo de contraer enfermedades tales como trastornos cardiovasculares, diabetes mellitus, obesidad, hiperlipidemia, cáncer, entre otras [3, 4], ya que son fuente de una gran variedad de antioxidantes naturales [5, 6, 7], que pueden ser utilizados para disminuir la oxidación de productos cárnicos [5, 8].

Los productos cítricos son fuentes importantes de aceite esenciales, carotenoides y compuestos fenólicos, que los convierte en recursos atractivos en la valoración agroindustrial como fuente importante de aditivos en la industria alimentaria $[9,10], \sin$ embargo los compuestos fenólicos tienden a formar radicales libres, pero gracias a su capacidad antioxidante estos compuestos, inhiben la producción de radicales libres [11, 12], que a su vez proporcionan efectos beneficiosos en la salud humana, ya que estos pueden ayudar en el daño hepático y gástrico sufrido por la ingesta excesiva de alcohol y a la protección del intestino [13], es así como el presente estudio pretende evaluar la capacidad de inhibición de los compuestos fenólicos presentes en Citrus sinensis mediante la de degradación de un sustrato como el beta caroteno para ser aplicado en un producto cárnico embutido y así mismo conocer la posible aceptación sensorial.

Es así como el presente estudio pretende evaluar la capacidad de inhibición de los compuestos fenólicos presentes en Citrus sinensis mediante la de degradación de un sustrato como el beta caroteno para ser aplicado en un producto cárnico embutido y así mismo conocer la posible aceptación sensorial.

\section{Materiales y métodos}

El desarrollo de la investigación se dio en cinco etapas importantes, la primera etapa comprendió la adquisición y preparación del material, como segunda etapa, se llevó el secado del material vegetal, en una tercera fase se realizaron las pruebas de extracción mediante los métodos ultrasonido y el método de Soxhlet, como cuarta fase se realizó la determinación de los compuestos fenólicos por análisis por cromatografía, por último se realizó el análisis de la actividad antioxidante mediante la utilización de la solución $\beta$-caroteno, con la aplicación en un embutido cárnico y posterior análisis sensorial para la aceptación del producto.

La muestra tomada fue de $5,7 \mathrm{~kg}$ de naranja valencia de la cual se obtuvo $1500 \mathrm{~g}$ de material vegetal fresco (cascara de Citrus sinensis) a la cual se le realizó una adecuación para su procesamiento.

\subsection{Obtención de harina de la cáscara de $\mathrm{C}$. Sinensis}

Se lavaron las cascaras con agua, adecuándose en pedazos de $0.5 \mathrm{~cm}$ por $0.5 \mathrm{~cm}$ hasta lograr un peso equivalente [14]. Posteriormente se pesaron, y se secaron a temperatura ambiente para su molienda, siendo esta de tipo artesanal [15], utilizando para ello un molino de granos tradicional corona, con el cual se obtuvo la harina. de la cual se generaron partículas con tamaños entre 250 y $850 \mu \mathrm{m}$.

\subsection{Extracción de compuestos fenólicos utilizando dos métodos}

Se realizaron las pruebas de extracción evaluando los métodos ultrasonido y el método de Soxhlet, con la finalidad de buscar el mejor rendimiento para la obtención del aceite. Para la extracción con ultrasonido se colocaron; $50 \mathrm{~g}$ de cáscara y $250 \mathrm{~mL}$ de etanol al $96 \%$ en un Beaker el cual se introdujo en un sonicador Branson 1800 serie CPXH en una relación 1:5 a temperatura de 25 ${ }^{\circ}$ C. En cuanto a la extracción por Soxhlet, se colocaron $10 \mathrm{~g}$ de harina de cáscara de naranja en los cartuchos del equipo, para lo cual se midieron $200 \mathrm{~mL}$ de etanol al 96\% en una relación 1:20, (10 g de harina y $200 \mathrm{~mL}$ de etanol), el cual se colocó en un balón a una temperatura de 25080 
${ }^{\circ} \mathrm{C}$, con un volumen inicial total de etanol de 400 $\mathrm{mL}$ realizándose dos repeticiones, posteriormente los extractos alcohólicos obtenidos se pasaron por evaporador rotatorio para concentrar las muestras obtenidas.

\subsection{Determinación de los compuestos fenólicos}

La determinación de compuestos fenólicos se realizó por cromatografía de alta eficiencia, en un cromatógrafo liquido (LC) Agilent Technologies 1200 Series (Palo Alto, California, EE.UU.), con un detector UVVis de arreglo de diodos (HPLC/DAD), a $\lambda=245$ $\mathrm{nm}$, La columna empleada en el análisis fue KINETEX (C18) (Phenomenex), $100 \mathrm{~mm} \times 4.6 \mathrm{~mm}$ (d.i.) x $2.6 \mu \mathrm{m}$ (tamaño de partícula), La inyección se realizó en modo automático (Viny $=10 \mu \mathrm{L}$ ), con gradiente de elución como se muestra en la Tabla 1. Para este análisis se tomó como referencia xantinas cafeína (Part $\mathrm{N}^{\circ}$ C8960-250G, Sigma-Aldrich), teobromina (Part $\mathrm{N}^{\circ}$ T4500-25G, Sigma-Aldrich) y teofilina (Part $\mathrm{N}^{\circ} \mathrm{T} 1633-25 \mathrm{G}$, Sigma-Aldrich); las catequinas $\left( \pm\right.$ )- catequina (C) (Part N ${ }^{\circ}$ C1788-500MG, SigmaAldrich), (-)-epigalocatequina galato (EGCG) (Part $\mathrm{N}^{\circ}$ E4143-50MG, Sigma-Aldrich), (-)-epicatequina (EC) (Part $N^{\circ}$ E1753-1G, Sigma-Aldrich), (-)epicatequina galato (ECG) (Part $\mathrm{N}^{\circ}$ E3893-10MG, Sigma-Aldrich), (-)- epigalocatequina (EGC) (Part $\mathrm{N}^{\circ}$ E3768-5MG, Sigma-Aldrich); los flavonoides ácido caféico (Part $\mathrm{N}^{\circ} \mathrm{C} 0625$, Sigma-Aldrich), acido p-cumárico (Part $\mathrm{N}^{\circ} \mathrm{C} 9008$, Sigma-Aldrich), ácido rosmarínico (Part $\mathrm{N}^{\circ}$ 536954-5G, SigmaAldrich), quercetina (Part $N^{\circ}$ Q4951-10G, SigmaAldrich), naringenina (Part $N^{\circ}$ N5893-1G, SigmaAldrich), luteolina (Part N ${ }^{\circ}$ L9283-10MG, SigmaAldrich), kaempferol (Part N ${ }^{\circ}$ K0133-50MG, SigmaAldrich), ácido ursólico (Part $\mathrm{N}^{\circ}$ U6753-100MG, Sigma-Aldrich), pinocembrina (Part $\mathrm{N}^{\circ}$ P5239, Sigma-Aldrich), ácido carnósico (Part N ${ }^{\circ}$ C060910MG, Sigma-Aldrich) y apigenina (Part $\mathrm{N}^{\circ} \mathrm{A} 3145$ 25MG, Sigma-Aldrich) a un flujo de $1 \mathrm{~mL} / \mathrm{min}$ y temperatura de $35^{\circ} \mathrm{C}$.

\subsection{Determinación de la actividad antioxidante}

Un indicador importante para conocer las bondades antioxidantes de frutas y vegetales es el secuestro de radicales libres por polifenoles [16, 17], en
Tabla 1. Gradiente de elución empleado en la metodología para el análisis cualitativo simultaneo de 24 compuestos antioxidantes

\begin{tabular}{ccc}
\hline$t(\min )$ & $\begin{array}{c}\text { \% Solvente A (ácido } \\
\text { acético } 0.3 \%)\end{array}$ & $\begin{array}{c}\text { \% Solvente D (acetonitrilo } \\
\text { HPLC) }\end{array}$ \\
\hline 0 & 95,5 & 4,5 \\
13 & 95,5 & 4,5 \\
14 & 85,0 & 15 \\
17 & 85,0 & 15 \\
20 & 78,0 & 22 \\
28 & 78,0 & 22 \\
30 & 0 & 100 \\
33 & 0 & 100 \\
34 & 95,5 & 4,5 \\
40 & 95,5 & 4,5 \\
\hline
\end{tabular}

este caso la actividad antioxidante se trabajó de acuerdo a la metodología de propuesta por [18], para lo cual se tomó $1 \mathrm{~mL}$ de solución de $\beta$ caroteno $(0.4 \mathrm{mg} / \mathrm{mL}$ en cloroformo) evaporando hasta sequedad posteriormente se agregaron 0.02 $\mathrm{mL}$ de ácido linoleico, $0.2 \mathrm{~mL}$ de Tween $20 \mathrm{y}$ $0.2 \mathrm{~mL}$ de los extractos crudos, agregándose 20 $\mathrm{mL}$ de agua oxigenada al 5\%, se determinó su absorbancia a $470 \mathrm{~nm}$, posterior a ello, las muestras se sometieron autooxidación térmica en baño a 50 ${ }^{\circ} \mathrm{C}$, leyendo su absorbancia cada 10 minutos durante 120 minutos, preparando un estándar con ácido ascórbico y un control con metanol al $80 \%$, además de un blanco con agua destilada. Para la evaluación de la actividad antioxidante en los extractos de la cáscara de naranja valencia se utilizó el método de decoloración del $\beta$-caroteno, en el cual, el valor de la actividad antioxidante se calculó teniendo en cuenta la ecuación propuesta por [19], como un porcentaje de inhibición relativa para el control, la actividad antioxidante (AA).

$$
\begin{gathered}
A A=\frac{\left(R_{\text {control }}-R_{\text {mиеstral }}\right)}{R_{\text {control }}} * 100 \\
R=\left(\operatorname{In} \frac{A 0}{A t}\right) / t
\end{gathered}
$$

Tomando a $R$ como la velocidad de degradación de la muestra control, $A 0$ como la absorbancia de la muestra control en el tiempo cero y At como absorbancia de la muestra control en el tiempo $t$. 


\section{Resultados Y Discusiones}

Del proceso de molienda seca realizada, se obtuvo la harina con partículas con tamaños entre 250 y $850 \mu \mathrm{m}$, lo que permite facilitar la extracción de constituyentes como aceites [20].

\subsection{Extracción por ultrasonido}

Para ello se realizaron tres extracciones, utilizando la relación 1:5 (50 g de cascara y $250 \mathrm{~mL}$ de etanol), con un volumen total inicial de $750 \mathrm{~mL}$ en etanol empleado, después de la sonicación y filtrado, para lo cual se obtuvo un volumen total de $610 \mathrm{~mL}$, con un porcentaje de rendimiento del $81.3 \% \mathrm{~m} / \mathrm{v}$, este método se caracteriza por la agitación por ondas sonoras a través de un disolvente aumentando la presión que provoca una mejor penetración del disolvente al interior del sólido [21].

\subsection{Extracción por Soxhlet}

Al realizar la evaluación de los métodos se observó que el método de extracción Soxhlet obtuvo rendimiento de $89,5 \%, \mathrm{~m} / \mathrm{v}$ en comparación con el ultrasonido el cual obtuvo un $81,3 \% \mathrm{~m} / \mathrm{v}$ en rendimiento, que en relación a su absorbancia, la utilización de mayor cantidad de harina en ultrasonido permitió mayor absorción de etanol, con lo que se estableció una diferencia del $8.2 \%$ menos en relación a la extracción por Soxhlet lo que permitió mayor favorabilidad con este último.

\subsection{Determinación cualitativa de compuestos fenólicos o antioxidantes}

Para la determinación cualitativa de los compuestos fenólicos en las muestras se realizó la comparación de los tiempos de retención, $t_{R}$ los cuales se encuentran en la Tabla 2 y los espectros UVVis, utilizando una solución patrón de los ácidos fenólicos, xantinas, catequinas y flavonoides en una concentración conocida.

Las muestras de extracto C. sinensis obtenida por ultrasonido y Soxhlet, fueron analizadas por duplicado, las figuras 1 y 2 se observa el análisis obtenido por cromatografía de los extractos de cascara de naranja.
Tabla 2. Tiempos de retención de ácidos fenólicos

\begin{tabular}{lc}
\hline Compuesto & $t_{R} / \mathrm{min}$ \\
\hline Ácido gálico & 1.99 \\
Teobromina & 3.64 \\
Teofilina & 5.73 \\
Acido -hidroxibenzoico & 6.84 \\
epigalocatequina & 9.17 \\
catequina & 9.17 \\
Ácido vanilico & 10.98 \\
Ácido cafeico & 12.20 \\
cafeína & 12.20 \\
epicatequina & 16.53 \\
Epigaloatequina galato & 16.88 \\
Acido -cumarico & 17.17 \\
Ácido felurico & 18.67 \\
Epicatequina galato & 19.73 \\
Ácido rosmarinico & 22.01 \\
Ácido trans-cinamico & 25.23 \\
Quercetina & 26.86 \\
Luteolina & 27.19 \\
Naringenina & 30.45 \\
Apigenina & 30.62 \\
kaempferol & 30.65 \\
Pinocembrina & 31.12 \\
Ácido carmosico & 31.62 \\
Ácido ursolinico & 31.81 \\
\hline & \\
&
\end{tabular}

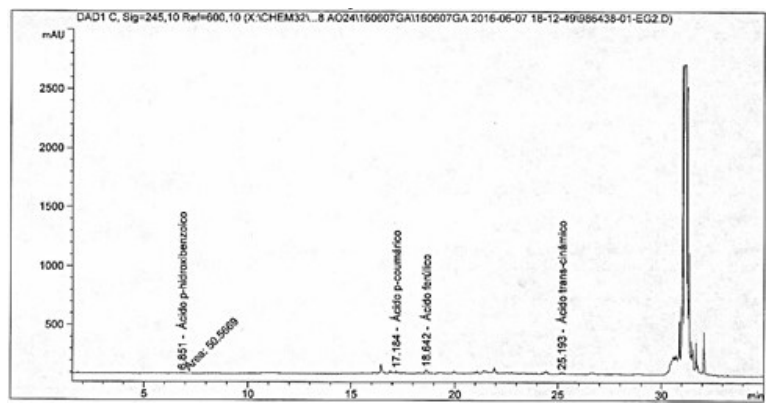

Figura 1. Extracción por ultrasonido en etanol relación $1: 5$.

En la Tablas 3 y 4 se observan los tiempos de retención y área de los picos principales del cromatograma, del extracto de cascara de naranja.

Los compuestos fenólicos determinados mediante la extracción por ultrasonido tratamiento T1 y extracción por Soxhlet tratamiento T2 se reportan a continuación en la Tabla 5. 


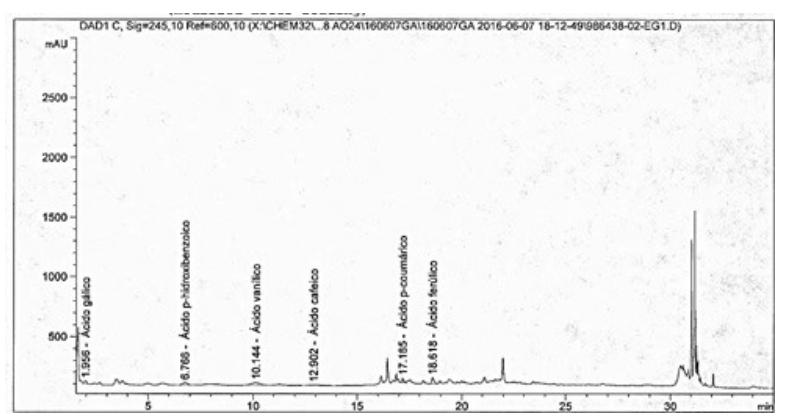

Figura 2. Extracción por Soxhlet en etanol relación $1: 20$.

Tabla 3. Extractos obtenidos por ultrasonido

\begin{tabular}{cccc}
\hline $\begin{array}{c}\text { Tiempo de } \\
\text { retención }(\mathbf{m i n})\end{array}$ & Área $[\mathbf{m A U} * \mathbf{S}]$ & Norm \% & Nombre \\
\hline 6.829 & 49.44165 & 1.970688 & Ácido p-hidrobenzoico \\
17.183 & 82.54964 & 46.773747 & Ácido p-coumárico \\
18.641 & 317.29688 & 33.999813 & Ácido ferúlico \\
25.212 & 122.35406 & 17.255751 & Ácido trans-cinámico \\
\hline
\end{tabular}

Tabla 4. Extractos obtenidos por Soxhlet

\begin{tabular}{cccc}
\hline $\begin{array}{c}\text { Tiempo de } \\
\text { retención (min) }\end{array}$ & Área [mAU*S] & Norm \% & Nombre \\
\hline 1.956 & 205.71246 & 17.943403 & Ácido gálico \\
6.766 & 376.80294 & 4.934656 & Ácido p-hidroxibenzoico \\
10.144 & 705.99133 & 31.940691 & Ácido vanílico \\
12.902 & 172.15836 & 3.807234 & Ácido caféico \\
17.185 & 143.43559 & 26.703120 & Ácido p-coumárico \\
18.618 & 416.70419 & 14.670897 & Ácido ferúlico \\
\hline
\end{tabular}

Tabla 5. Ácidos fenólicos determinados por HPLC/DAD, a $\lambda=245 \mathrm{~nm}$.

\begin{tabular}{ll}
\hline Identificación de la muestra & Compuestos detectados \\
\hline $\begin{array}{ll}\text { T1, 1:5 harina de cascara de } & \text { Acido } p \text {-hidroxibenzoico e Ácido } p \text { - } \\
\text { naranja y etanol, extracción } & \text { cumaricoÁcido felurico Ácido trans- } \\
\text { por ultrasonido } & \text { cinamico }\end{array}$ \\
\hline
\end{tabular}

T2, 1:20 harina de cascara de Ácido galico Ácido p-hidroxibenzoico naranja y etanol, extracción Ácido vanilico Ácido cafeico Ácido $p$ por Soxhlet cumarico Ácido felurico

La mayoría de los compuestos fenólicos presentes en la naranja incluyen ácidos hidroxicinámicos (HCA) y flavonoides, entre los cuales flavanones son los más frecuentes [22], Cabe mencionar que la combinación de la acción de diversos polifenoles, con lleva a que estos actúen como antioxidantes, protegiendo a la salud humana [23, 24], sin embargo, el contenido el contenido de estos compuestos fenólicos puede variar dependiendo la variedad del fruto [25].

\subsection{Actividad antioxidante}

La evaluación de la actividad antioxidante (AA) reporto mayor porcentaje en el extracto de naranja valencia obtenido por Soxhlet, con respecto al extracto obtenido con ultrasonido y el estándar (ácido ascórbico), alcanzando un valor porcentual de 91, 333\%, como se evidencia en la Tabla 6.

Los antioxidantes analizados se mantuvieron en rangos similares con el método de decoloración del $\beta$-caroteno como se puede observar en la figura 3, obteniéndose la mayor y menor capacidad antioxidante en los extractos de la naranja valencia, indicando que el método de extracción influye directamente en la capacidad inhibidora de oxidación, siendo el método de extracción por Soxhlet, el de mayor eficiencia oxidante.

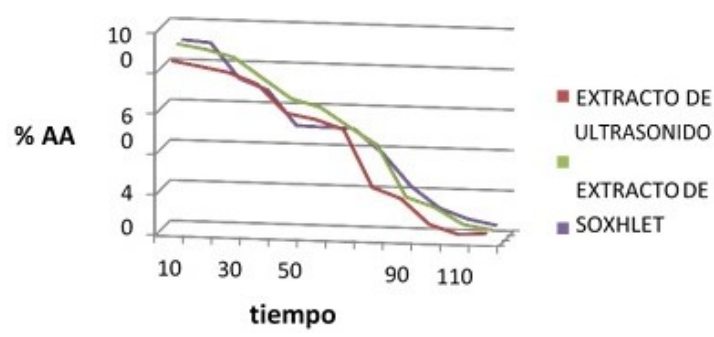

Figura 3. Actividad antioxidante de los extractos de la naranja valencia y el ácido ascórbico por el método de decoloración del $\beta$-caroteno.

El beta caroteno decolora rápidamente sin la presencia de un antioxidante, en caso contrario este neutraliza los radicales libres formados [26], lo que demuestra que, sin duda, la cáscara de cítricos son una fuente importante de antioxidantes naturales, pues las cáscaras tienen alta actividad antioxidante, pudiendo resultar un mejor sustituto, en lugar de los antioxidantes sintéticos, ya que este permiten alargar la vida útil de los productos alimenticios [27].

En cuanto a la aplicación de los compuestos fenólicos (Acido p-hidroxibenzoico, Ácido vanilico,Ácido cafeico,Acido p-cumarico Ácido felurico) se realizó en un producto embutido crudo y fresco, teniendo en cuenta que son las condiciones más factibles en la producción de peroxidación lipídica de las 
Tabla 6. Actividad antioxidante por decoloración del $\beta$-caroteno, medido a 470 nm

\begin{tabular}{cccccccc}
\hline \multirow{2}{*}{ Tiempo $(\mathrm{min})$} & \multicolumn{2}{c}{ Extracto de ultrasonido } & \multicolumn{2}{c}{ Extracto de Soxhlet } & \multicolumn{2}{c}{ Ácido ascorbico (estándar) } & \multicolumn{2}{c}{ Metanol (control) } \\
\cline { 2 - 7 } & Absorbancia & \%AA & Absorbancia & \%AA & Absorbancia & \%AA & Absorbancia \\
\hline 0 & 1,387 & & 1,348 & & 1,160 & & 1,236 \\
10 & 1,334 & 85,486 & 1,317 & 91,333 & 1,132 & 90,898 & 0,945 \\
20 & 1,324 & 83,019 & 1,308 & 88,996 & 1,128 & 89,781 & 0,94 \\
30 & 1,285 & 80,464 & 1,273 & 85,359 & 1,042 & 72,563 & 0,836 \\
40 & 1,280 & 75,043 & 1,246 & 75,540 & 1,044 & 67,248 & 0,896 \\
50 & 1,234 & 61,272 & 1,216 & 65,853 & 0,998 & 50,159 & 0,914 \\
60 & 1,176 & 58,683 & 1,159 & 62,178 & 0,949 & 49,734 & 0,829 \\
70 & 1,109 & 54,128 & 1,072 & 53,018 & 0,907 & 49,545 & 0,759 \\
80 & 1,022 & 26,017 & 1,071 & 44,272 & 0,898 & 37,979 & 0,818 \\
90 & 0,983 & 20,857 & 0,948 & 19,079 & 0,826 & 21,939 & 0,800 \\
100 & 0,926 & 8,445 & 0,924 & 14,418 & 0,785 & 11,512 & 0,795 \\
110 & 0,849 & 4,064 & 0,835 & 6,389 & 0,719 & 6,512 & 0,741 \\
120 & 0,857 & 4,896 & 0,830 & 4,207 & 0,713 & 3,863 & 0,745 \\
\hline
\end{tabular}

grasas, lo que puede causar la oxidación y aparición de olores y sabores [28], para ello se aplicaron en tres prototipos en un producto cárnico, variando el antioxidante, inicialmente se empleó como aditivo antioxidante, ácido ascórbico en una muestra control y dos más en muestras en las que se utilizó como antioxidantes los compuestos fenólicos extraídos de la cascara de naranja valencia, en una proporción de $0.01 \%$ en masa, posteriormente se realizó un análisis sensorial a treinta personas a que se les presentaron tres muestras enumeradas las cuales fueron clasificadas en un escala sensorial como muy rancio, rancio, neutro, fresco, muy fresco. el análisis sensorial se empleó una escala para determinar el grado de rancidez de cada muestra.

Para la evaluación estadística se utilizó el paquete SPSS y se aplicó una prueba $\mathrm{T}$ (análisis de factor), donde se le asignó un valor a cada termino, en el que 1 equivale a muy rancio, 2 a rancio, 3 a neutro, 4 a fresco y 5 a muy fresco, en la Tabla 7 se aprecian los resultados obtenidos.

El análisis estadístico proporciono resultados que permitieron demostrar que para las tres muestras el promedio se encontró entre el rango neutro y fresco. Al comparar las muestras de antioxidante extraído de la cascara dela naranja valencia con respecto a la muestra control en la cual se empleó como antioxidante ácido ascórbico, el producto embutido
Tabla 7. Resultados del panel sensorial

\begin{tabular}{lccc}
\hline & \multicolumn{3}{c}{ Muestras } \\
\cline { 2 - 4 } Escala & 4587 & 8261 & 6455 \\
\hline Muy rancio & 0 & 1 & 1 \\
Rancio & 1 & 1 & 3 \\
Neutro & 18 & 15 & 17 \\
Fresco & 7 & 7 & 6 \\
Muy fresco & 4 & 6 & 3 \\
Total & 30 & 30 & 30 \\
\hline
\end{tabular}

con el extracto antioxidante obtenido por ultrasonido presento una diferencia promedio de 0,0666 a favor, mientras que en la muestra con antioxidante obtenido por Soxhlet fue de 0,23334 por debajo del control, como se evidencia en la Tabla 8.

Lo que demuestra que las variables son independientes desde el punto de vista estadístico, ya que su valor está por encima del rango 0,05 , lo cual indica que no existe diferencia significativa entre las tres muestras de los productos embutidos.

\section{Conclusiones}

El análisis por cromatografía permitió identificar la presencia de cinco compuestos fenólicos por cada método utilizado, que mediante la decoloración del $\beta$-caroteno lo cual indican que los compuestos extraídos por los métodos ultrasonido y Soxhlet 
Tabla 8. Análisis del coeficiente de confianza de la prueba

\begin{tabular}{|c|c|c|c|c|c|c|}
\hline & \multirow[b]{2}{*}{$t$} & \multirow[b]{2}{*}{$\mathrm{gl}$} & \multirow[b]{2}{*}{ DSig. (bilateral) } & \multirow[b]{2}{*}{ Diferencia de medias } & \multicolumn{2}{|c|}{$\begin{array}{l}\text { 95\% intervalo de confianza para la } \\
\text { diferencia }\end{array}$} \\
\hline & & & & & Inferior & Superior \\
\hline $\begin{array}{l}\text { Muestra con antioxidante ácido } \\
\text { ascorbico }\end{array}$ & ,000 & 29 & 1,000 & ,00000 &,- 2898 & ,2898 \\
\hline $\begin{array}{l}\text { Muestra con antioxidante obtenido } \\
\text { por ultrasonido }\end{array}$ & ,37529 & 29 & ,710 & ,06666 &,- 2967 & ,4301 \\
\hline $\begin{array}{l}\text { Muestra con antioxidante obtenido } \\
\text { por Soxhlet }\end{array}$ & $-1,424$ & 29 & , 165 &,- 23334 &,- 5685 & , 1018 \\
\hline
\end{tabular}

de la cascara de naranja variedad valencia, tiene una alta capacidad de inhibir la oxidación de un sustrato, comprobando que el método de extracción por Soxhlet es más eficiente, pues su rendimiento fue mayor en relación al método de ultrasonido, lo cual demuestra que existe una correlación entre la cantidad de compuestos extraídos y la capacidad antioxidante, permitiendo de esta manera determinar que el material vegetal de $C$. sinecis posee atributos que pueden ser aprovechados para su empleo en la industria cárnica, lo cual no afectaría las propiedades organolépticas de los productos elaborados.

\section{Agradecimientos}

A la Universidad Francisco de Paula Santander por su valiosa colaboración.

\section{Referencias}

[1] León Fierro, L. G., Rodriguez-Villalobos, J. M., Candia-Luján, R., Carrasco-Legleu, C. E., and Enríquez del Castillo, L. A. (2019). Efectividad de los suplementos antioxidantes en la mejoría del desempeño físico atlético. Artículo de revisión. Revista Habanera de Ciencias Médicas, vol. 18 no. 2 pp. 194-216.

[2] Sánchez-Valle, V., and Méndez-Sánchez, N. (2018). Estrés oxidativo, antioxidantes y enfermedad. Médica Sur, vol. 20 no. 3 pp. 161-168.

[3] George, SM, Park, Y., Leitzmann, MF, Freedman, ND, Dowling, EC, Reedy, J., ... y Subar, AF (2009). Ingesta de frutas y verduras y riesgo de cáncer: un estudio de cohorte prospectivo. La Revista Estadounidense De Nutrición Clínica, vol. 89, no. 1 pp. 347-353.

[4] Reddy, KS y Katan, MB (2004). Dieta, nutrición y prevención de hipertensión y enfermedades cardiovasculares. Nutrición de Salud Pública, vol. 7 no. 1a pp. 167-186.

[5] Ahmad, S. R., Gokulakrishnan, P., Giriprasad, R., and Yatoo, M. A. (2015). Fruit-based natural antioxidants in meat and meat products: A review. Critical reviews in food science and nutrition, vol. 55 no. 11 pp. 1503-1513.

[6] Karre, L., Lopez, K., and Getty, K. J. (2013). Natural antioxidants in meat and poultryproducts. Meat Science, vol. 94 no. 2 pp. 220-227.

[7] Speisky, H., López-Alarcón, C., Gómez, M., Fuentes, J. y Sandoval-Acuña, C. (2012). Primera base de datos basada en web sobre fenólicos totales y capacidad de absorción de radicales de oxígeno (ORAC) de frutas producidas y consumidas dentro de la región de los Andes del sur de América del Sur. Revista de Química Agrícola Alimentaria, vol. 60 no. 36 pp. 8851-8859.

[8] Falowo, AB, Fayemi, PO y Muchenje, V. (2014). Antioxidantes naturales contra el deterioro oxidativo de lípidos y proteínas en carne y productos cárnicos: una revisión. Food Research International, vol. 64, 171-181.

[9] M'hiri, N., Ioannou, I., Ghoul, M., and Mihoubi Boudhrioua, N. (2017). Phytochemical characteristics of citrus peel and effect of conventional and nonconventional processing 
on phenolic compounds: A review. Food Reviews International, vol. 33 no. 6 pp. 587 619.

[10] Sanz-Puig, M., Moreno, P., Pina-Pérez, M. C., Rodrigo, D., and Martínez, A. (2017). Combined effect of high hydrostatic pressure (HHP) and antimicrobial from agro-industrial by-products against S. Typhimurium. $L W T$, vol. $77,126-133$.

[11] Quideau, S., Deffieux, D., Douat-Casassus, C. y Pouysegu, L. (2011). Polifenoles vegetales:propiedades químicas, actividades biológicas y síntesis. Angewandte Chemie Edición Internacional, vol. 50 no. 3 pp. $586-$ 621.

[12] Shahidi, F., and Ambigaipalan, P. (2015). Phenolics and polyphenolics in foods, beverages and spices: Antioxidant activity and health effects-A review. Journal of Functional Foods, vol. 18, 820-897.

[13] Quiñones, M., Miguel, M., and Aleixandre, A. (2012). Los polifenoles, compuestos de origen natural con efectos saludables sobre el sistema cardiovascular. Nutrición Hospitalaria, vol. 27 no. 1 pp. 76-89.

[14] Castro-Vazquez, L., Alañón, M. E., Rodríguez-Robledo, V., Pérez-Coello, M. S., Hermosín Gutierrez, I., Díaz-Maroto, M. C., ... and Arroyo-Jimenez, M. D. M. (2016). Bioactive flavonoids, antioxidant behaviour, and cytoprotective effects of dried grapefruit peels (Citrus paradise Macf.). Oxidative Medicine and Cellular Longevity, 2016.

[15] Tovar, C. D. G., and Colonia, B. S. O. (2013). Producción y procesamiento del maíz en Colombia. Revista Guillermo de Ockham, vol. 11 no. 1 pp. 97-110.

[16] Maraldi, T., Vauzour, D. y Angeloni, C. (2014). Polifenoles dietéticos y sus efectos en la bioquímica y fisiopatología celular 2013.

[17] Arteaga, A., and Arteaga, H. (2016). Optimización de la capacidad antioxidante, con- tenido de antocianinas y capacidad de rehidratación en polvo de arándano (Vaccinium corymbosum) microencapsulado con mezclas de hidrocoloides. Scientia Agropecuaria, vol. 7 (SPE pp. 191-200.

[18] Miller, N. J., Sampson, J., Candeias, L. P., Bramley, P. M., and Rice-Evans, C. A. (1996). Antioxidant activities of carotenes and xanthophylls. FEBS Letters, vol. 384 no. 3 pp. 240-242.

[19] Al-Saikhan, M. S., Howard, L. R., and Miller Jr, J. C. (1995). Antioxidant activity and total phenolics in different genotypes of potato (Solanum tuberosum, L.). Journal of Food Science, vol. 60 no. 2 pp. 341-343

[20] Castelló Gómez, M. L., Barrera Puigdollers, M. C., Pérez Esteve, E., and Betoret Valls, N. (2017). Reducción del tamaño de partícula y tamizado de partículas.

[21] Park, J. H., Lee, M., and Park, E. (2014). Antioxidant activity of orange flesh and peel extracted with various solvents. Preventive Nutrition and Food Science, vol. 19 no. 4 pp. 291.

[22] García Alonso, S., Pérez Pastor, R. M., Sevillano Castano, M. L., and Garcia Frutos, F. J. (2010). Analytical Evaluation to Determine Selected PAHs in a Contaminated Soil With Type II Fuel; Método Optimizado de Extracción por Ultrasonidos para la Determinación de PAHs Seleccionados en un Suelo Contaminado con Fuel de Tipo II.

[23] Parinandi, N. L., Maulik, N., Thirunavukkarasu, M., and McFadden, D. W. (2015). Antioxidants in longevity and medicine 2014.

[24] Grosso, G., Galvano, F., Mistretta, A., Marventano, S., Nolfo, F., Calabrese, G., ... and Scuderi, A. (2013). Red orange: experimental models and epidemiological evidence of its benefits on human health. Oxidative medicine and cellular longevity, 2013. 
[25] Pérez-Nájera, V. C., Lugo-Cervantes, E. C., Gutiérrez-Lomelí, M., and Del-Toro-Sánchez, C. L. (2013). Extracción de compuestos fenólicos de la cáscara de lima (Citrus limetta Risso) y determinación de su actividad antioxidante. Biotecnia, vol. 15 no. 3 pp. 1822.

[26] Kang, HJ, Chawla, SP, Jo, C., Kwon, JH y Byun, MW (2006). Estudios sobre el desarrollo de polvo funcional a partir de piel de cítricos. Tecnología de Fuentes Biológicas, vol. 97 no. 4 pp. 614-620.
[27] Ordoñez-Gómez, E. S., Reátegui-Díaz, D., and Villanueva-Tiburcio, J. E. (2018). Polifenoles totales y capacidad antioxidante en cáscara y hojas de doce cítricos. Scientia Agropecuaria, vol. 9 no. 1 pp. 113-121.

[28] Zhang, W., Xiao, S., and Ahn, D. U. (2013). Protein oxidation: basic principles and implications for meat quality. Critical Reviews in Food Science and Nutrition, vol. 53 no. 11 pp. 1191-1201. 